\title{
DC-coupled burst-mode receiver with high sensitivity, wide dynamic range and short settling time for symmetric 10G-GPONs
}

\author{
Jasmien Put, ${ }^{1, *}$ Xin Yin, ${ }^{1}$ Xing-Zhi Qiu, ${ }^{1}$ Jan Gillis, ${ }^{1}$ Jochen Verbrugghe, ${ }^{1}$ Johan \\ Bauwelinck, ${ }^{1}$ Jan Vandewege, ${ }^{1}$ Fabrice Blache, ${ }^{2}$ Delphine Lanteri, ${ }^{2}$ Mohand Achouche, ${ }^{2}$ \\ Heinz-Georg Krimmel, ${ }^{3}$ Dora van Veen, ${ }^{4}$ and Peter Vetter ${ }^{4}$ \\ ${ }^{I}$ Ghent University, INTEC/IMEC, Sint-Pietersnieuwstraat 41, 9000 Ghent, Belgium \\ ${ }^{2} I I I-V$ Lab, a joint lab of Alcatel-Lucent Bell Labs, Thales R\&T and CEA Leti, Route de Nozay, 91460 Marcoussis, \\ France \\ ${ }^{3}$ Alcatel-Lucent, Bell Laboratories, Lorenzstr. 10, 70435 Stuttgart, Germany \\ ${ }^{4}$ Alcatel-Lucent, Bell Laboratories, 600 Mountain Ave, Murray Hill, New Jersey 07974, USA \\ jasmien.put@intec.ugent.be
}

\begin{abstract}
This paper presents a high performance DC-coupled $10 \mathrm{Gbit} / \mathrm{s}$ APD-based optical burst-mode receiver (BM-RX) for symmetric 10GGPONs. The BM-RX can handle upstream bursts with a loud/soft range wider than $24.0 \mathrm{~dB}$, and the measured sensitivity is $-30.0 \mathrm{dBm}$ at $\mathrm{BER}=$ $10^{-3}$ after a start of burst settling time no longer than $51 \mathrm{~ns}$. This paper also investigates, for the first time, the inter-burst guard time required for a 10 Gbit/s BM-RX with auto-reset generation, while the PON operates upstream at a high raw BER and is using FEC.
\end{abstract}

(C)2011 Optical Society of America

OCIS codes: (060.2360) Fiber optics links and subsystems; (060.0060) Fiber optics and optical communications.

\section{References and links}

1. S. Takahashi, K. Shiba, E. Mizuki, K. Makita, and A. Tajima, "Over 25-dB dynamic range 10-/1-Gbps optical burst-mode receiver using high-power-tolerant APD," Proceedings of Conference on Optical Fiber Communication, Vol. 1-5, pp. 616-618 (2009).

2. J. Sugawa, D. Mashimo, and H. Ikeda, "10.3Gbps burst-mode receiver capable of upstream transmission with short overhead for 10G-EPON," 36th European Conference and Exhibition on Optical Communication (ECOC), Mo.2.B.4 (2010).

3. M. Nakamura, S. Nishihara, T. Ito, T. Kurosaki, M. Nogawa, and Y. Ohtomo, "Burst-mode optical receiver ICs for broadband access networks," IEEE Bipolar/BiCMOS Circuits and Technology Meeting (BCTM), pp. 21-28 (2010).

4. K. Hara, S. Kimura, H. Nakamura, N. Yoshimoto, and H. Hadama, "Ultra Fast Response AC-Coupled BurstMode Receiver with High Sensitivity and Wide Dynamic Range for 10G-EPON System," IEICE Trans. Commun E 94-B, 1845-1852 (2011).

5. M. Noda, S. Yoshima, K. Ishii, S. Shirai, M. Nogami, and J. Nakagawa, "Dual-rate optical transceiver incorporating fully optimized burst-mode AGC/ATC functions for 10G-EPON systems," 36th European Conference and Exhibition on Optical Communication (ECOC), Mo.2.B.2 (2010).

6. X.-Z. Qiu, C. Melange, T. De Ridder, B. Baekelandt, J. Bauwelinck, X. Yin and J. Vandewege, "Evolution of burst mode receivers," 35th European Conference on Optical Communication (ECOC), 7.5.1. (2009).

7. C. Melange, X. Yin, B. Baekelandt, T. De Ridder, X.-Z. Qiu, J. Bauwelinck, J. Gillis, P. Demuytere and J. Vandewege, "Fully DC-coupled 10Gb/s burst-mode PON prototypes and upstream experiments with 58ns overhead," Optical Fiber Communication (OFC), OWX2 (2010).

8. T. De Ridder, P. Ossieur, C. Melange, B. Baekelandt, J. Bauwelinck, X.-Z. Qiu, and J. Vandewege, "10 Gbit/s burst-mode post-amplifier with automatic reset," Electron. Lett. 44(23), 1371-1373 (2008).

9. X. Yin, X.-Z. Qiu, J. Gillis, J. Put, J. Verbrugghe, J. Bauwelinck, J. Vandewege, F. Blache, D. Lanteri, M. Achouce, H. Krimmel, D. van Veen, and P. Vetter, "DC-Coupled Burst-Mode Receiver with High Sensitivity, Wide Dynamic Range and Short Settling Time for Symmetric 10G-GPONs," 37th European Conference and Exhibition on Optical Communication (ECOC), Mo.1.C.5. (2011).

10. M. Lahrichi, E. Derouin, D. Carpentier, N. Lagay, J. Decobert, G. Glastre, and M. Achouche, "Very Low Dark Current AlInAs/GaInAs SAGM Avalanche Photodiodes for 10Gb/s applications," 35th European Conference and Exhibition on Optical Communication (ECOC), 9.2.2 (2009). 
11. J. Put, X. Yin, J. Gillis, X.-Z. Qiu, J. Bauwelinck, J. Vandewege, H.-G. Krimmel, and P. Vetter, "10 Gbit/s burstmode limiting amplifier with switched time constants for fast settling and large CID tolerance," Electron. Lett. 47(17), 970-972 (2011).

12. X. Yin, J. Put, J. Verbrugghe, J. Gillis, X.-Z. Qiu, J. Bauwelinck, J. Vandewege, H.-G. Krimmel, and M. Achouche, "A 10Gb/s Burst-Mode TIA with On-Chip Reset/Lock CM Signaling Detection and Limiting Amplifier with a 75ns Settling Time, Solid-State Circuits Conference Digest of Technical Papers (ISSCC), (to be published).

\section{Introduction}

As the demand for broadband services keeps rising, interests in time division multiplexing (TDM) passive optical networks (PONs) continue to grow. Anticipating an explosive growth of the worldwide data traffic, IEEE 10GE-PON and ITU-T XG-PON1 systems have been standardized and start being deployed. The 10GE-PON and the XG-PON1 can provide respectively 10/10 Gbit/s and 10/2.5 Gbit/s downstream/upstream transmission capacities. For the near future a symmetric 10/10G-GPON with shorter overhead may provide higher network transmission capacity and efficiency, especially when short bursts are emitted upstream.

The optical line termination (OLT) of the 10G-GPON relies on a high-performance burstmode receiver (BM-RX). To cover the high optical power budget required for co-existence with already deployed PONs, avalanche photodiode (APD) burst-mode receivers (BM-RX) are used with strong forward error correction (FEC). Several $10 \mathrm{Gbit} / \mathrm{s}$ BM-RXs have been published [1-4] for IEEE 10GE-PONs, where the BM-RX settling time requirement is quite relaxed. The development of a $10 \mathrm{Gbit} / \mathrm{s}$ BM-RX for advanced 10G-GPONs however remains challenging due to the combined requirements: high BM-RX sensitivity $(<-28 \mathrm{dBm})$, wide dynamic range $(\mathrm{DR}>22 \mathrm{~dB})$ and short settling time $(<100 \mathrm{~ns})$ are the BM-RX's key figures of merit.

Most $10 \mathrm{Gbit} / \mathrm{s}$ BM-RX designs to date use AC-coupled interfaces [1-4]. However, a fundamental limit on performance of AC-coupled BM-RXs results from the AC-coupling time constant tradeoff. As shown in Fig. 1., the coupling capacitance between the burst-mode transimpedance amplifier (BM-TIA) and the burst-mode limiting amplifier (BM-LA) should be chosen carefully to speed-up the settling time while tolerating a long sequence of consecutive identical digits (CID). Therefore, AC-coupling requires line coding, such as $8 \mathrm{~B} / 10 \mathrm{~B}$ and $64 \mathrm{~B} / 66 \mathrm{~B}$ used in $1 \mathrm{G}-\mathrm{EPON}$ and $10 \mathrm{G}-\mathrm{EPON}$ respectively, to limit the maximum CID length. The AC-coupling time constant tradeoff makes it difficult to achieve a response time below $400 \mathrm{~ns}$. One method to reduce the response time below $400 \mathrm{~ns}$, while maintaining operational conditions with long CIDs, is to use two-stage feedback inside the BM-LA [3]. In [4], an AC-coupled BM-RX applies baseline wander common mode rejection combined with inverted distortion techniques, resulting in a settling time of $150 \mathrm{~ns}$ at the cost of an additional BM-Rx sensitivity penalty of $1 \mathrm{~dB}$.
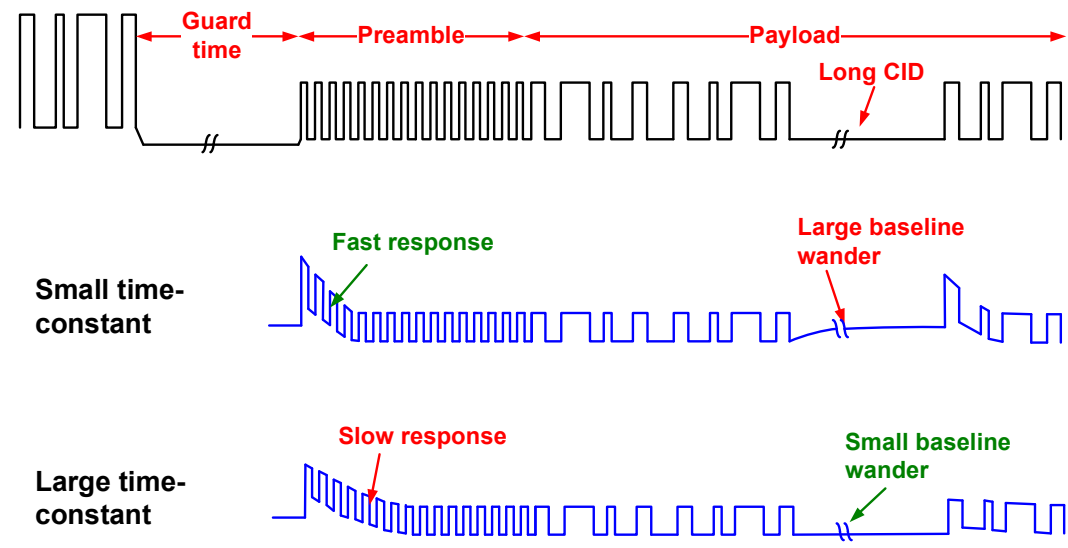

Fig. 1. Time constant tradeoff in AC-coupled BM-RX. 
The alternative is a DC-coupled interface between a BM-TIA and a BM-LA. Ref [5] presents a DC-coupled receiver which meets the 10G-EPON specifications with a settling time of $800 \mathrm{~ns}$. As there is no coupling time constant tradeoff, a DC-coupled BM-RX can achieve a much shorter settling time [6]. Our previous work shows a $10 \mathrm{Gbit} / \mathrm{s}$ DC-coupled PIN-Based BM-RX for a $10 \mathrm{Gbit} / \mathrm{s}$ long reach PON [7]. The upstream channels were OSNR limited by using optical amplification, and the BM-RX did not require high sensitivity or wide dynamic range. Therefore threshold extraction by peak detection was chosen to obtain a short overhead of $58 \mathrm{~ns}$, and a measured BM-RX sensitivity of $-10.9 \mathrm{dBm}$ at a BER of $10^{-10}$, with a loud/soft ratio of $10.6 \mathrm{~dB}$. However, a peak-detection based feed-forward circuit is inherently not very accurate, and results in relatively high power consumption. In this paper we present a new DC-coupled feedback BM-RX which combines the advantages of DC- and AC-coupled approaches to provide an overall optimized performance.

Efficient PON uplink transmission requires a DC-coupled BM-RX with a short overhead for any incoming burst, irrespective of the received signal strength. The burst overhead time is a combination of inter-burst guard time and BM-RX settling time. A fast settling DC-coupled $\mathrm{BM}-\mathrm{RX}$ needs a reset signal at the end of each burst, in order to erase all the information of the previous burst and to prepare the BM-RX for the next burst to come. This reset signal may originate from the medium access control (MAC) layer of the PON, as there the respective timeslots for upstream transmission are allocated, but good system practice avoids timecritical signals crossing the boundary between the PON physical layer and the MAC layer. At $10 \mathrm{Gbit} / \mathrm{s}$, the guard time is substantially longer than the maximum CID sequence that can occur in the burst payload, so one can distinguish the long logic 0 of the guard time from the CIDs in the burst payload of the incoming bit stream. As a result, the BM-RX is able to detect the end-of-burst (EoB) itself, and to automatically generate a reset signal without the need for time-critical signals from the MAC layer. Our research group published a $10 \mathrm{Gbit} / \mathrm{s}$ DCcoupled BM-RX with an auto-reset function in [8]. As EoB detection might fail with noisy incoming signals, this paper investigates the robustness of the auto-reset generation at high bit error rate (BER), a situation that occurs when feedforward error correction (FEC) is deployed.

This paper is an expanded version of our presentation on ECOC 2011 [9]. The different building blocks of the BM-RX, namely the APD, BM-TIA and BM-LA, are discussed in more detail in Section 2. In Section 3, the influence of a high pre-FEC BER on the BM-RX auto-reset function is studied for the first time. Section 4 presents the measurement setup and experimental results, similar to [9].

\section{10 Gbit/s DC-coupled BM-RX}

Figure 2 depicts the $10 \mathrm{Gbit} / \mathrm{s}$ DC-coupled BM-RX configuration. It contains an APD, a BMTIA and a BM-LA, which are described in detail below. The DC-coupled BM-TIA and BMLA chips were fabricated in a STMicroelectronics $0.13 \mu \mathrm{m}$ SiGe BiCMOS process.

\#155804 - \$15.00 USD

(C) 2011 OSA
Received 30 Sep 2011; revised 3 Nov 2011; accepted 3 Nov 2011; published 1 Dec 2011

12 December 2011 / Vol. 19, No. 26 / OPTICS EXPRESS B596 


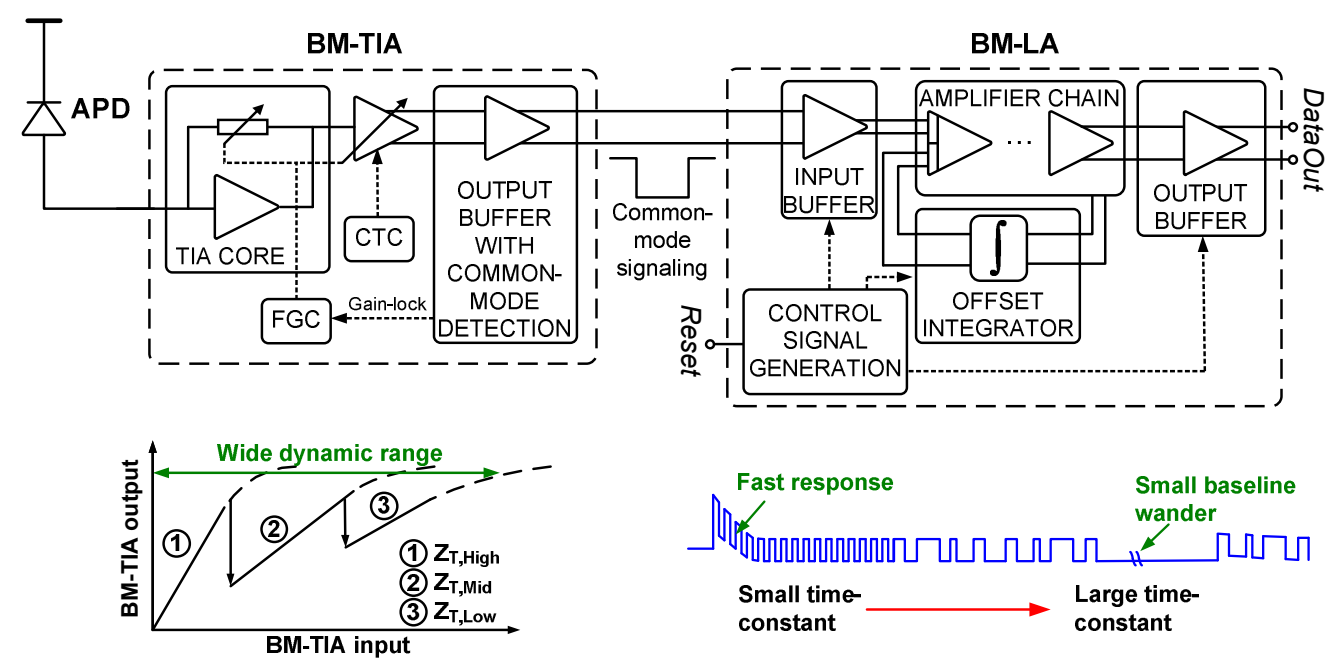

Fig. 2. The simplified block diagram of the $10 \mathrm{Gbit} / \mathrm{s}$ DC-coupled BM-RX.

\subsection{The $10 \mathrm{Gbit} / \mathrm{s}$ BM APD-TIA}

The $10 \mathrm{Gbit} / \mathrm{s}$ BM APD-TIA converts the input current from a 10G APD photodiode into a differential output voltage. Low noise amplification and high overload handling are the main figures of merit. The APD used for this work is an AlInAs/InGaAs APD structure with an excess noise factor $\mathrm{F}(\mathrm{M})$ of 3.3 at $\mathrm{M}=10$, as shown in Fig. 3a. Figure $3 \mathrm{~b}$ illustrates that a $3-\mathrm{dB}$ bandwidth of about $10 \mathrm{GHz}$ is achieved for avalanche gains between 4 and 15 , and that the extrapolated gain-bandwidth product is about $150-160 \mathrm{GHz}[10]$.

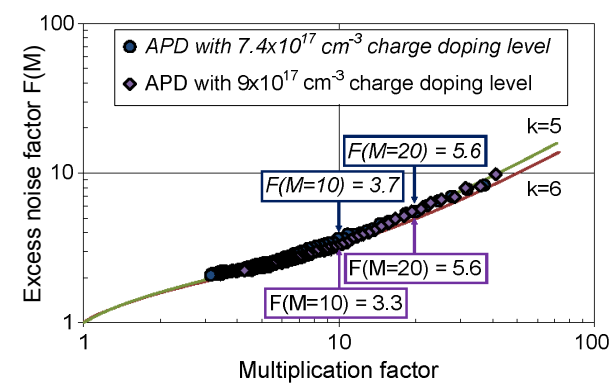

(a)

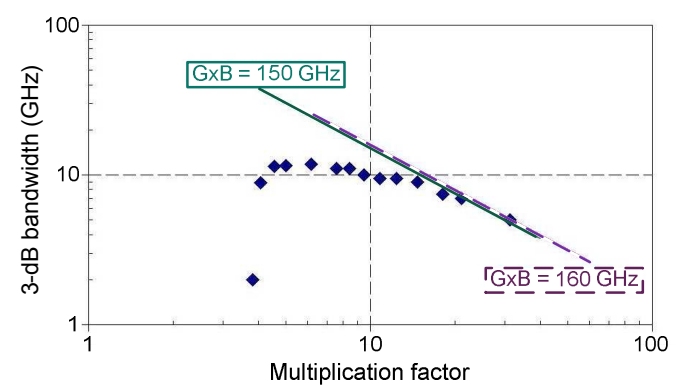

(b)

Fig. 3. (a) Excess noise factor versus multiplication gain of APDs with two doping levels. (b) 3 -dB Bandwidth versus multiplication gain of AlInAs/InGaAs APD with $9 \times 1017 \mathrm{~cm}^{-3}$ charge doping level.

The $10 \mathrm{Gbit} / \mathrm{s}$ BM-TIA incorporates a fast gain control (FGC) and gain-locking function. It achieves a wide dynamic range by three transimpedance gain settings. When a burst has ended, the BM-TIA is reset to a high transimpedance gain $\mathrm{Z}_{\mathrm{T}, \mathrm{High}}(65 \mathrm{~dB} \Omega)$ for maximum $\mathrm{RX}$ sensitivity. The single-ended $\mathrm{O} / \mathrm{E}$ response at high gain $\mathrm{Z}_{\mathrm{T}, \mathrm{High}}$ is measured at TIA's output $\mathrm{P}$ using HF probes (Fig. 4) and it shows $\sim 6 \mathrm{GHz} 3 \mathrm{~dB}$ bandwidth. The power of the new incoming burst is measured at the output of the TIA core. As soon as this power exceeds a certain power level, the transimpedance is switched from $\mathrm{Z}_{\mathrm{T}, \mathrm{High}}$ to a middle gain setting of $\mathrm{Z}_{\mathrm{T}, \mathrm{Mid}}(48 \mathrm{~dB} \Omega$ ) by switching the feedback resistance of the TIA core. A second gain switching from $Z_{\mathrm{T}, \text { Mid }}$ to the low gain $Z_{\mathrm{T}, \text { Low }}(44 \mathrm{~dB} \Omega)$ is set by changing the gain of the single-to-differential convertor, and happens when the received signal power exceeds a second power threshold. This three-step gain switching is accomplished within several nanoseconds. After the gain switching process, and within the burst preamble, the TIA 
transimpedance gain is locked in order to avoid bit error rate (BER) degradation due to $Z_{T}$ switching inside the burst payload interval. The BM-TIA also performs a coarse threshold compensation (CTC) process, which sets the balance signal of the single-ended to differential converter according to the transimpedance gain settings. This minimizes the DC offset in the TIA output signal at the RX sensitivity level, and helps reduce the settling time of the BMLA. Figure 2 indicates that the BM-TIA has no reset pin. The reset signal is conveyed from the BM-LA via a common-mode signaling method. The input buffer of the BM-LA alters the common-mode voltage of the BM-TIA output. The BM-TIA senses these common mode changes, so that no dedicated reset signal line is required.

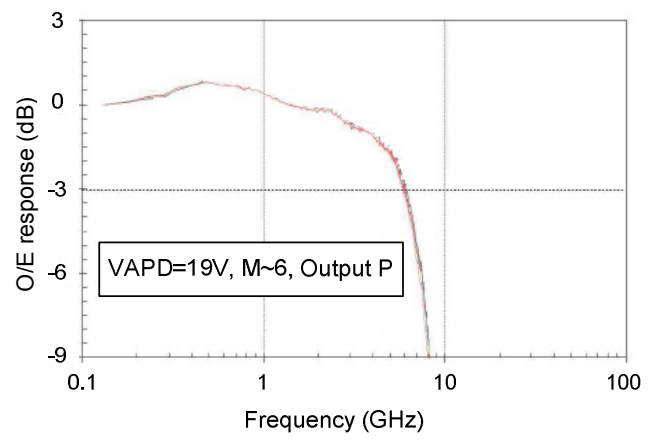

Fig. 4. O/E response of the $10 \mathrm{Gbit} / \mathrm{s}$ APD-TIA module using HF probes.

\subsection{The $10 \mathrm{Gbit} / \mathrm{s} B M-L A$}

The subsequent $10 \mathrm{Gbit} / \mathrm{s}$ feedback type BM-LA [11] has two operation modes: a fast decision threshold level detection mode and a slow threshold level tracking mode. When a new burst arrives, the threshold detection circuit first performs fast offset compensation and amplitude recovery. At the end of the preamble, the BM-LA switches to the slow tracking mode, which is crucial to provide a higher tolerance to CIDs. The time constant of the offset compensation loop is set by the bias current of the offset integrator, which is implemented as a low-frequency amplifier with large capacitive loads. The bias current switches between respectively $1 \mathrm{~mA}$ and $15 \mu \mathrm{A}$ for the fast and slow threshold extraction. At the end of the burst, the BM-LA resets the decision threshold to the default state, by discharging the capacitive loads of the offset integrator, and waits for the arrival of a next burst. Therefore, a reset signal is needed for the BM-LA.

The advantage of using two operation modes in the BM-LA is verified by means of simulations. We have simulated the RX settling time and output jitter in the presence of long CIDs for both a BM-LA with one fixed operation mode and for the new BM-LA with two operation modes. A fixed $2^{11}-1$ PRBS data pattern with inserted 64-, 128-, 256- and 512-bit CIDs was applied in the transient simulation. By using a smaller time constant at the start of each burst, the new design shows $\sim 75 \%$ reduction of the settling time, from $200 \mathrm{~ns}$ to $41 \mathrm{~ns}$. This significant settling time reduction does not deteriorate the CID tolerance. As shown in Fig. 5, the proposed $10 \mathrm{Gbit} / \mathrm{s}$ BM-LA can handle up to 512-bit of CID by switching the time constant to a larger value. In this way, the proposed BM-RX can achieve simultaneously a fast response and a large CID tolerance. The interested reader can find more details about the implementation of the BM-LA in our previous published paper [11]. 


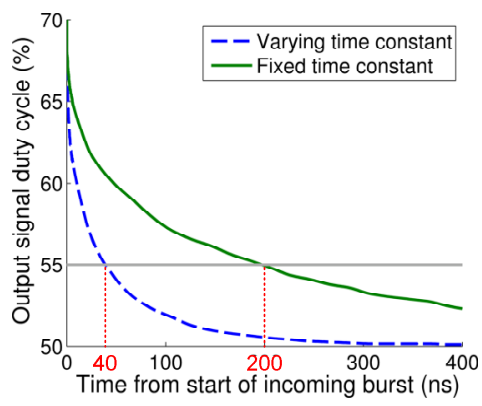

(a)

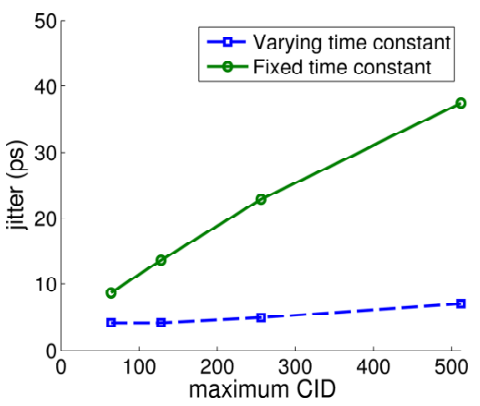

(b)

Fig. 5. (a) Duty cycle distortion during preamble for $300 \mathrm{mVpp}$ with $150 \mathrm{mV}$ offset, (b) Data dependent output jitter for $20 \mathrm{mVpp}$ input with $10 \mathrm{mV}$ offset

\section{Auto-reset generation at high BER}

In order to guarantee high sensitivity and short overhead time, the transimpedance gain setting of the BM-TIA and the threshold of the BM-LA are reset at the end of the burst. Therefore, an external reset signal is needed. However, when the guard time can be distinguished logically from the maximum CID of the payload, the BM-RX preferably generates the reset signal itself after detecting the EoB. In this way, no time-critical signals must cross the boundary between the MAC layer and the physical layer [6]. The reset generator, published by our research group in [8], detects the EoB when the BM-LA receives a long sequence of logic 0's without any logic 1 . This duration is chosen sufficiently longer than the maximum CID in order to avoid an untimely reset signal during the payload. However, the auto-reset generation will suffer from noise, especially at the high pre-FEC BER. Figure 6 illustrates the influence of high BER in case of [8], where a reset signal is generated after counting $n$ consecutive 0-bits. Every 1-bit that is erroneously received during the guard time causes the counter to restart, resulting in a delayed reset generation. When the time needed to detect the EoB is longer than the guard time, no reset signal is generated. Consequently, the BM-RX might miss the next burst. Hence, the minimum guard time should be chosen carefully.

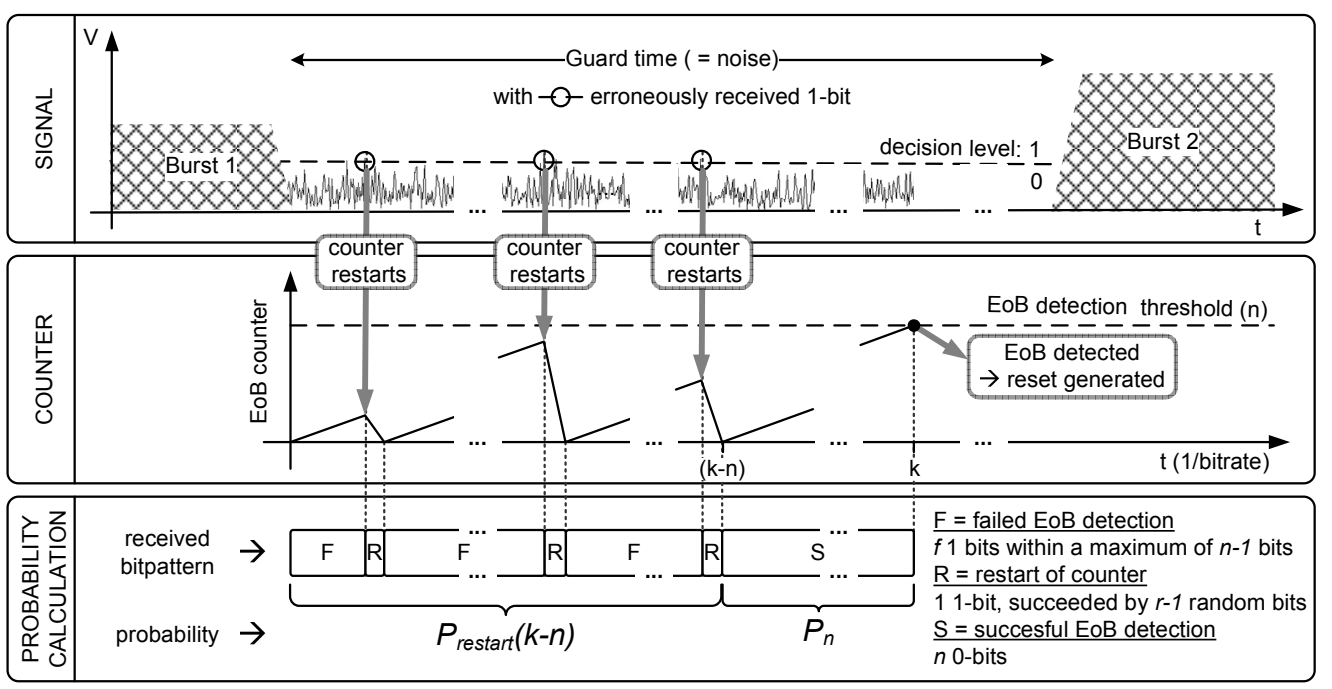

Fig. 6. Auto-reset generation at high BER scenario.

Therefore the probability of detecting the EoB is calculated versus the guard time. Two probabilities, namely $P_{n}$ and $P_{\text {restart }}$, are defined as illustrated in Fig. 6. $P_{n}$ is the probability of detecting the EoB, or in other words receiving $n$ consecutive 0-bits during the guard time, and

\#155804 - \$15.00 USD

(C) 2011 OSA 
is given by $P_{n}=(1-B E R)^{n} . P_{\text {restart }}(k)$ on the other hand is the probability that the counter is restarted after exactly $k$ bits. This happens when the $k^{\text {th }}$ bit is erroneously detected as a logic 1 and EoB was not detected before. Thus $P_{\text {restart }}(k)$ can be calculated as:

$$
P_{\text {restart }}(k)=\left(1-P_{E O B}(k-1)\right) \cdot B E R
$$

With $P_{E o B}(k)$ the probability of detecting EoB within $k$ bits. Consequently, the probability of detecting EoB after exactly $k$ bits is given by $P_{\text {restart }}(k-n) \cdot P_{n}$ and thus the probability of detecting EoB within $k$ bits after the end of the preceding burst is equal to:

$$
P_{E o B}(k)=\left\{\begin{array}{cc}
0 & k<n \\
P_{n} & k=n \\
P_{E o B}(k-1)+P_{\text {restart }}(k-n) \cdot P_{n} & k>n
\end{array}\right.
$$

Figure 7 shows the probability of missing the EoB, thus missing a reset, versus the guard time at $10 \mathrm{Gbit} / \mathrm{s}$ for different BERs in case $n$ is chosen equal to 100. Allowing a missed reset in only 1 out of $10^{10}$ cases requires a minimum guard time of $20 \mathrm{~ns}, 43 \mathrm{~ns}$ and $69 \mathrm{~ns}$ at a BER of respectively $10^{-10}, 10^{-4}$ and $10^{-3}$. The sudden drops of the curve are due to the small chance of restarting the counters, which is equal to the BER. Note that these simple calculations don't take into account the time needed to restart the counters.

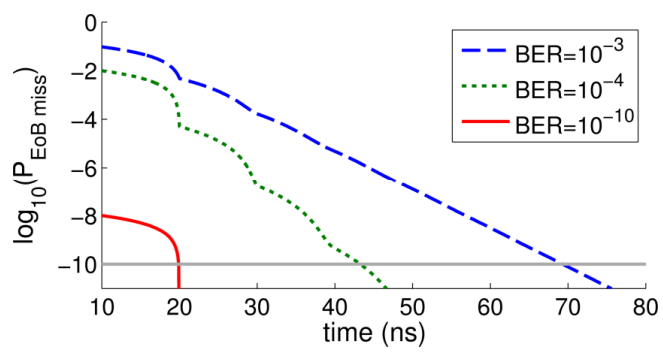

Fig. 7. Probability of missing a reset during the guard time for different BERs (when $n=100$ ).

To make the auto-reset generation more robust at high BER, we can further extend the technique used in [8]. Instead of restarting the counter after a 1-bit fault, we could tolerate a number $f$ faults before the counter restarts again. The idea is to generate a reset signal after receiving $n$ consecutive bits, including a maximum of $f$ 1-bits. In other words, the EoB detection only restarts the counter after receiving $f+1$ 1-bits.

Formula (2) is still valid for evaluating this technique analytically. $P_{n}$ is now given by $P_{n}=\sum_{f^{\prime}=0}^{f^{\prime}=f} C_{n}^{f^{\prime}}(1-B E R)^{n-f^{\prime}} B E R^{f^{\prime}}$. Taking into account the time needed to restart the counter, expressed as $r$ bit periods, $P_{\text {restart }}$ is equal to:

$$
P_{\text {restart }}(k)=\left\{\begin{array}{cc} 
& k=0 \\
1 & 0<k<f+r+n \\
0 & k \geq f+r+n \\
\sum_{\text {NoR }=\left[\frac{k}{n+r-1}\right\rfloor}^{\text {NoR }\left\lfloor\frac{k}{f+r}\right\rfloor} \operatorname{Div}(k-r \cdot N o R, N o R) \cdot B E R^{\text {NoR. }(f+1)} \cdot(1-B E R)^{k-N o R(r+f)} &
\end{array}\right.
$$

Where NoR is the number of times the counter restarts during the EoB-detection. Each restart of the counter is preceded by a sequence of maximally $n-1$ bits, containing $f 1$-bits (as can be seen in the third part of Fig. 6). Div $(k, p l)$ is thus the number of possible divisions of $k$ bits over $p l$ places with $f$ ones and maximally $n-l$ bits in each place and is given by: 


$$
\operatorname{Div}(k, p l)=\left\{\begin{array}{cc}
C_{k}^{f} & p l=1 \\
\sum_{k^{\prime}=\max (f, k-(p l-1) \cdot(n-1))}^{k^{\prime}=\min (n-1, k-(p l-1) \cdot} C_{k^{\prime}}^{f} \cdot \operatorname{Div}\left(k-k^{\prime}, p l-1\right) & p l>1
\end{array}\right.
$$

Figure 8 shows the probability of missing the EoB versus the guard time at $10 \mathrm{Gbit} / \mathrm{s}$ and BER $=10^{-4}$, assuming that the counters are restarted within $2 \mathrm{~ns}$. In order to avoid a wrongly generated reset during the payload, $n$ is set to respectively 100,155 and 215 for $f$ equal to 0,1 and 2. As $P_{n}$ decreases with increasing $f$, the three curves overlap each other. As $n$ is taken larger to avoid a false reset generation, the actual EoB detection takes more time. Furthermore, the chance of restarting the counter reduces with increasing $f$, as it is now equal to $B E R^{f+1}$, resulting in a stepped curve as shown in Fig. 8. The required guard time, allowing a missed reset in only one out of $10^{10}$ cases, is respectively $51 \mathrm{~ns}, 50 \mathrm{~ns}$ and $45 \mathrm{~ns}$. When allowing 0 bit errors, an extra $7 \mathrm{~ns}$ is needed, due to the restart time of the counter. This restart time has less influence when more bit errors are allowed during the detection. Furthermore, the probability of a false reset generation during payload decreases for higher $f$, e.g. a 215-bit long sequence with only 21 -bits is unlikely to occur during payload. Consequently, the new proposed reset generation is more robust.

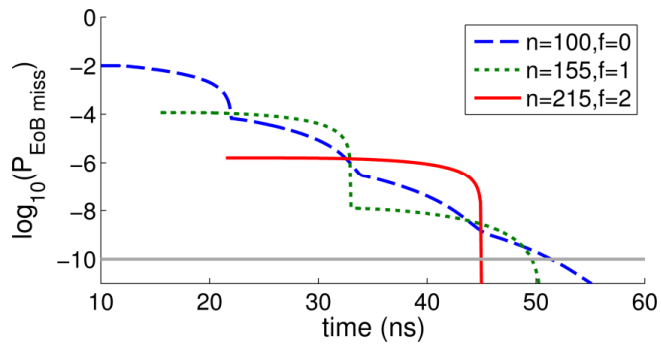

Fig. 8. Probability of missing a reset during the guard time for different settings at $B E R=10^{-4}$.

This newly proposed auto-reset generator was successfully implemented in the $2^{\text {nd }}$ version of the BM-LA IC, using a clock counter and a 1-bit counter. Reset is generated after the clock counter exceeds the clock threshold $(n)$. Every time that the 1-bit counter exceeds its threshold $(f)$, the EoB detection is restarted. Both $n$ and $f$ are fully programmable, which makes the auto-reset generation flexible to use. The interested reader can find more details about the implementation of the auto-reset generation in our paper [12].

\section{Experimental setup and results}

The $10 \mathrm{Gbit} / \mathrm{s}$ BM-RX performance was evaluated using the experimental set-up shown in Fig. 9(a). Two $1.3 \mu \mathrm{m}$ burst-mode transmitters (BM-TXs) named TX \#1 and TX \#2, are alternately sending $10 \mathrm{Gbit} / \mathrm{s}$ burst packets. TX \#1 contains a $1.3 \mu \mathrm{m}$ electro-absorption modulator (EAM) integrated with a DFB-laser diode. It has an output power of $+4.4 \mathrm{dBm}$ and an extinction ratio (ER) of $10 \mathrm{~dB}$. The output optical power of the two BM-TXs can be adjusted by two variable optical attenuators (VOAs), namely VOA \#1 and VOA \#2 for respectively TX \#1 and TX \#2. For interference measurements on weak bursts preceded by strong bursts, the power level provided by TX \#2 is set at $-6 \mathrm{dBm}$ after a two-to-one $3 \mathrm{~dB}$ optical combiner, a level close to the APD overload, while only the BER of the bursts emitted by TX \#1 is recorded using an Agilent 81250 ParBERT. The $10 \mathrm{Gbit} / \mathrm{s}$ burst packets shown in Fig. 9(b) consist of a $51.2 \mathrm{~ns}$ preamble and a $1280 \mathrm{~ns}$ payload. The guard time between bursts is set to $25.6 \mathrm{~ns}$. The payload is composed of 3 sections of non-return-to-zero (NRZ) $2^{31}-1$ pseudo random bit sequence (PRBS) data patterns, separated by 2 sequences of 72 CIDs (respectively 72 logic 1's and logic 0's), in order to validate the BM-RX's tolerance to long CIDs. In this measurement setup the ParBERT also generates the reset signal. On-chip autoreset generation is successfully tested in a $2^{\text {nd }}$ version of the BM-LA IC, as will be presented in [12]. 


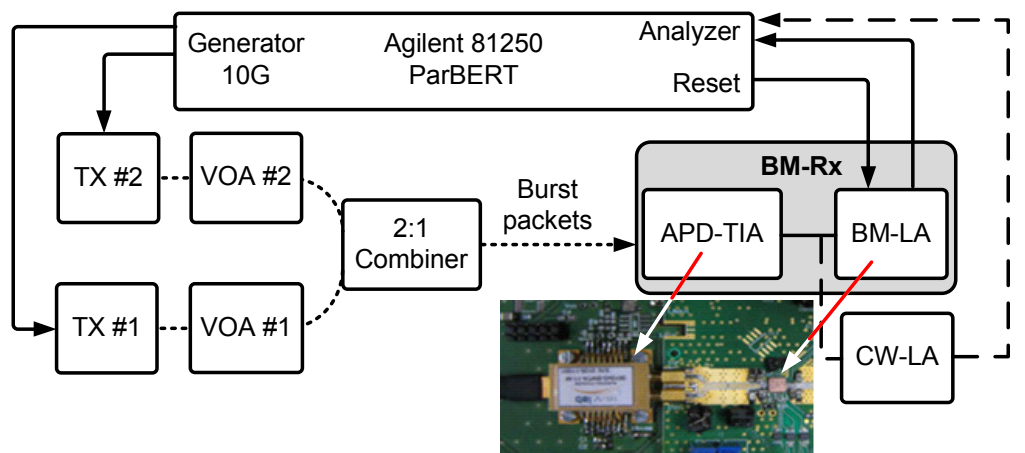

(a)

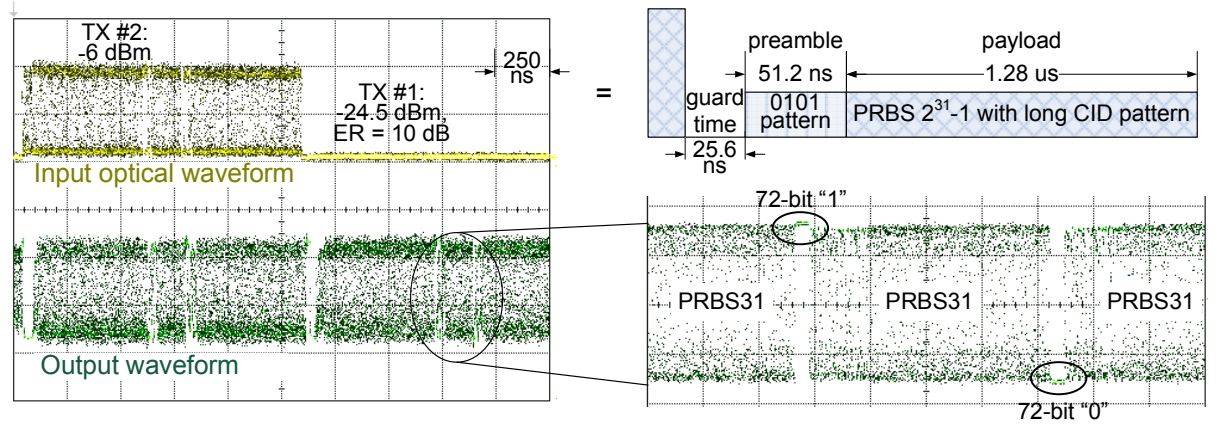

(b)

Fig. 9. The test setup and burst data patterns for the $10 \mathrm{Gbit} / \mathrm{s}$ upstream experiment.

The measured BER curves are displayed in Fig. 10. The input sensitivity of the BM-TIA with a continuous-mode $(\mathrm{CW}) \mathrm{LA}$ is $-31.9 \mathrm{dBm}$ at a pre-FEC BER of $10^{-3}$ and $-27.1 \mathrm{dBm}$ at a BER of $10^{-10}$ for CW input signals. In the BM-RX back-to-back (B2B) configuration, the measured sensitivity at a pre-FEC BER of $10^{-3}$ is $-30.8 \mathrm{dBm}$ and the sensitivity at a BER of $10^{-10}$ is $-26.0 \mathrm{dBm}$, mainly due to the input sensitivity penalty of the BM-LA. A new version BM-LA with improved sensitivity has been developed and manufactured at the time of writing. With the improved BM-LA sensitivity, we would expect the $10 \mathrm{Gbit} / \mathrm{s}$ BM-RX to show a better B2B sensitivity at a BER of $10^{-10}$. This will allow the advanced BM-RX to be used without FEC in networks that do not require maximum optical power budget. Thanks to the three transimpedance gain settings within the BM-TIA, the error free input overload level was found to be higher than $-5 \mathrm{dBm}$. This yields a dynamic range of more than $25.8 \mathrm{~dB}$.

In the experiments with 2 branches of BM-TXs, the BM-RX sensitivity measured on the weak burst emitted by TX \#1 is $-30.0 \mathrm{dBm}$ for the worst case when the output power of TX \#2 equals $-6 \mathrm{dBm}$. Thus the BM-RX penalty is $0.8 \mathrm{~dB}$ at a loud/soft ratio of $24.0 \mathrm{~dB}$. The BM-RX penalty at a BER of $10^{-10}$ was found to be even smaller, and about $0.35 \mathrm{~dB}$. From Fig. 10 (a) one can see that the $51 \mathrm{~ns}, 76 \mathrm{~ns}$ and $102 \mathrm{~ns}$ preamble performance is almost identical. A very smooth degradation is observed for the $25 \mathrm{~ns}$ preamble. Table 1 summarizes the performance evaluation of the $10 \mathrm{Gbit} / \mathrm{s}$ DC-coupled BM-RX. A pre-FEC BER threshold of $10^{-3}$ is employed as recommended for the upstream by IEEE 802.3av 10G-EPON. 


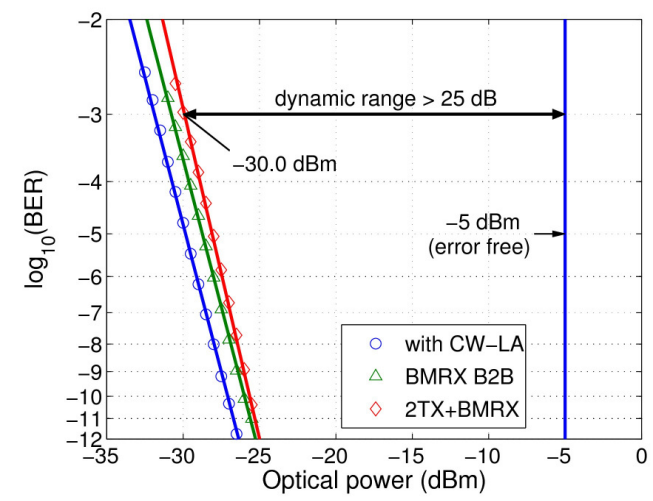

(a)

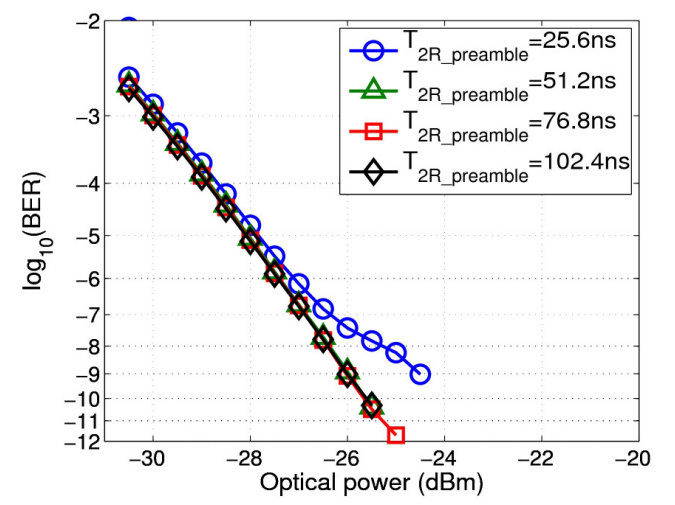

(b)

Fig. 10. (a) The measured BER curves for TIA+CW-LA, BM-RX B2B, and (b) 2 TX branches; BER vs. different preamble times (right).

Table 1. Performance Summary of the DC-coupled BM-RX at $10 \mathrm{Gbit} / \mathrm{s}$

\begin{tabular}{|l|c|c|c|c|}
\hline $\begin{array}{l}\text { 10 Gbit/s } \\
\text { Burst-mode }\end{array}$ & $\begin{array}{c}\text { With } \\
\text { CW-LA }\end{array}$ & $\begin{array}{c}\text { B2B } \\
\text { (TX + RX) }\end{array}$ & $\begin{array}{c}\text { BM-RX with } \\
2 \text { TX branches }\end{array}$ & $\begin{array}{c}\text { Test conditions: } \\
\text { DC-coupled NRZ data, without line } \\
\text { coding, }\end{array}$ \\
\hline \hline Sensitivity (dBm) & -31.9 & -30.8 & -30.0 & $\begin{array}{l}\text { 51 ns 2R RX settling time, 25 ns } \\
\text { guard time, } \\
\text { PRBS31 payload plus CID patterns } \\
\text { with 72x1's \& 72x0's } \\
\text { TX ER = 10 dB, external reset, APD } \\
\text { M }=10\end{array}$ \\
\hline Overload (dBm) & -5 & -5 & -6 & Loud/soft 24 \\
\cline { 1 - 2 }
\end{tabular}

\section{Conclusions}

A high performance DC-coupled $10 \mathrm{Gbit} / \mathrm{s}$ APD-based BM-RX, suitable for symmetric 10GGPONs, has been demonstrated with a RX sensitivity of $-30 \mathrm{dBm}$ at a BER of $10^{-3}$ while handling a loud/soft ratio of $24 \mathrm{~dB}$. The $10 \mathrm{Gbit} / \mathrm{s}$ BM-TIA applies a three-step fast gain setting, resulting in a high dynamic range of over $25 \mathrm{~dB}$. The DC offset in the LA's input signal is minimized at the RX sensitivity level by the CTC of the TIA. The residual offset is compensated within the BM-LA, using a feedback offset compensator with switched time constants. As a results, both a very short settling time of only $51 \mathrm{~ns}$ and a high tolerance to long sequences of CIDs is achieved.

This paper also presents a new method for the auto-reset generation, allowing one or more bit errors while detecting the EoB at high BERs. A thorough analytical study shows that the required guard time, allowing a missed reset in only one out of $10^{10}$ cases, is around $50 \mathrm{~ns}$.

\section{Acknowledgments}

This work was supported by the EU-funded FP7 ICT projects MARISE and EURO-FOS. The authors would like to thank STMicroelectronics for providing chipset fabrication, and Sumitomo Electric Device Innovations, Inc for providing 1.3 $\mu \mathrm{m}$ 10G EML devices. 